\title{
Evaluación de un modelo socio-cognitivo de la satisfacción laboral en trabajadores argentinos
}

Lucas Lapuente. Universidad Empresarial Siglo 21, Córdoba/Argentina

Pablo Ezequiel Flores Kanter. Universidad Empresarial Siglo 21, Córdoba/Argentina

Roger Muñoz Navarro. Universidad de Valencia/ España

Leonardo Adrián Medrano. Pontifícia Universidad Católica Madre y Maestra/Republica Dominicana

\section{Resumen}

Muchas teorías se han elaborado con el objeto de identificar los factores que permitan explicar la satisfacción laboral. Una de ellas es el Modelo Social Cognitivo de la Profesión propuesto por Lent. Este modelo propone integrar los factores afectivosdisposicionales y situacionales (condiciones laborales) con elementos socio-cognitivos y conductuales, los cuales afecten la satisfacción laboral. La presente investigación se propuso verificar el ajuste del modelo al contexto argentino por medio del análisis de ecuaciones estructurales. Las variables incluidas explican el 64\% de la Satisfacción Laboral. Asimismo, la mayoría de los path sugeridos por este modelo hacen una contribución significativa a la satisfacción, tanto directa como indirectamente. Considerando los resultados obtenidos pueden proponerse medidas preventivas, de promoción, o de solución para situaciones de insatisfacción laboral.

Palabras clave: satisfacción en el trabajo; trabajadores; desarrollo profesional.

\section{Resumo}

Avaliação de um modelo sócio-cognitivo da satisfação trabalhista em trabalhadores argentinos. Muitas teorias foram elaboradas com o objetivo de identificar os fatores explicativos da satisfação no trabalho. Uma delas é o Modelo Social Cognitivo da Profissão proposto por Lent. Este modelo propõe integrar os fatores afetivos disposicionais e situacionais (condições de trabalho) com elementos sócio-cognitivos e de conduta, os quais afetam a satisfação no trabalho. A presente investigação se propôs a verificar o ajuste do modelo ao contexto argentino mediante análise de equações estruturais. As variáveis incluídas explicam $64 \%$ da variância da satisfação no trabalho. Desse modo, a maioria dos caminhos sugeridos por este modelo oferecem uma contribuição significativa à satisfação, tanto direta como indiretamente. Os resultados obtidos permitem elaborar medidas preventivas, de promoção, ou de solução para situações de insatisfação no trabalho.

Palavras-chave: satisfação no trabalho; trabalhadores; desenvolvimento profissional.

\begin{abstract}
Evaluation of a socio-cognitive model of job satisfaction in Argentine workers. Many theories have been developed in order to identify the factors that explain job satisfaction. One of them is the Cognitive Social Model of Profession proposed by Lent. This model proposes to integrate affective-dispositional and situational factors (working conditions) with socio-cognitive and behavioural elements, which affect job satisfaction. The purpose of this investigation was to verify the adjustment of the model to the Argentinean context through the analysis of structural equations. The variables included explain $64 \%$ of the variability in labour satisfaction. Likewise, most of the paths suggested by this model make a significant contribution to satisfaction, both directly and indirectly. Considering the results obtained, preventive, promotional or solution measures can be proposed for situations of labour dissatisfaction.
\end{abstract}

Keywords: job satisfaction; workers; professional development. 
El estudio de los factores que contribuyen a mejorar el bienestar de las personas es un objetivo prioritario ya que contribuye a mejorar las relaciones interpersonales, el afrontamiento de problemas, la salud física, entre otros componentes saludables (Sanjuán Suárez \& Rueda Laffond, 2014). A su vez, uno de los factores que influyen sobre el nivel de bienestar subjetivo es la satisfacción laboral (Liu, Zhang, Chang, \& Wang, 2017; Spector, 1997).

La satisfacción laboral se define como un estado emocional positivo resultante de la interpretación de nuestra experiencia en el trabajo (Lent \& Brown, 2008). En otras palabras, la satisfacción con el trabajo puede definirse como el grado en que la persona está a gusto o le agrada su trabajo (Duffy \& Lent, 2009). En relación a este constructo, hace tiempo que los psicólogos organizacionales se encuentran interesados por comprender los factores responsables de la satisfacción laboral. Es decir, el interés se centra en conocer qué variables influyen sobre el grado en que las personas se sienten satisfechas, disfrutan, o les agrada su trabajo, ya sea este último entendido de manera global o respecto a una condición particular (Lent et al., 2011).

En este sentido, muchas investigaciones y teorías se han elaborado con el objeto de identificar los factores que permiten predecir o explicar la satisfacción laboral (Duffy \& Lent, 2009). Las mismas se han enfocado sobre diferentes predictores, como ser el ajuste entre la persona y el ambiente laboral, o las disposiciones individuales (e.g. rasgos de personalidad o afectivos; Lent \& Brown, 2006). Una de estas teorías es el Modelo Social Cognitivo de la Profesión propuesto por Lent (Lent et al. 2011). Este modelo parte de una visión socio-cognitiva y propone integrar a los factores afectivos-disposicionales y situacionales (condiciones laborales) elementos socio-cognitivos y conductuales, los cuales promuevan o reduzcan la satisfacción laboral (Lent \& Brown, 2006).

Sumado a lo anterior, el modelo social cognitivo de Lent se basa en una visión proactiva, de auto-direccionalidad, capturada por el concepto de agencia humana propuesta por Bandura (1999). La teoría percibe así a las personas como agentes activos capaces de direccionar su profesión, lo cual también se ve influenciado por el apoyo del ambiente y los recursos personales (Lent, 2013). El modelo se focaliza específicamente en los siguientes factores predictores de la satisfacción laboral: a) rasgos de personalidad/afectivos; b) participación en actividades dirigidas a metas; c) autoeficacia relacionada al trabajo; d) expectativas de resultado; e) obstáculos o apoyo del ambiente laboral. Finalmente, se mide el efecto conjunto de todas estas variables sobre f) la satisfacción general con la vida (Lent et al. 2011, figura 1).

Como describen Duffy y Lent (2009), hasta el momento existía evidencia acerca del efecto que tenían estas series variables de manera individual sobre la satisfacción laboral, pero escaseaban modelos que las integrasen. Así, por ejemplo, el estado afectivo o la tendencia a experimentar estados emocionales positivos, ha demostrado relaciones entre moderadas y fuertes con la satisfacción laboral. Por ejemplo, los rasgos afectivos positivos y negativos en cierto caso han demostrado explicar el $30 \%$ de la varianza de la satisfacción laboral (Lent \& Brown, 2006). Otro de los factores del modelo está relacionado a las metas, entendidas como la determinación a lograr cierto resultado o alcanzar cierto nivel de desempeño (Lent \& Brown, 2006). En este sentido, se ha podido verificar que tener metas valoradas y hacer progresos en éstas es un predictor importante del bienestar general (Duffy \& Lent, 2009; Lent $\&$ Brown, 2006). Otra variable considerada en el modelo es la creencia de autoeficacia, esto es, la creencia de que uno es capaz de llevar a cabo eficazmente una tarea o comportamiento específico. Se ha podido demostrar también que las creencias de autoeficacia permite predecir la satisfacción laboral de los trabajadores (Lent \& Brown, 2006). Otras de las variables consideradas por el modelo es el apoyo percibido, el cual refiere al grado en que los empleados se sienten apoyados por su ambiente laboral para llevar a cabo los objetivos relacionados al trabajo. Según Duffy y Lent (2009) el grado en que los empleados perciben apoyo de su trabajo se relaciona fuertemente con la satisfacción laboral experimentada. De manera similar, Lent y Brown (2008) dirán que la percepción de apoyo que la persona tenga en relación con su trabajo será una fuente importante de satisfacción laboral. La ultima variable considerada por este modelo y asociada a la satisfacción laboral son las expectativas de resultados (Lent \& Brown, 2006). En este sentido, Lent (2004) indicará que las expectativas de resultados tienen un efecto directo sobre los juicios de satisfacción laboral, pero también indirectos a través de la percepción del progreso en metas. Esta última relación se explica en función de que las personas constantemente anticipan las consecuencias de sus acciones, afectando esto en el establecimiento y ejecución de metas. 
La novedad del modelo social cognitivo de Lent se basa así en la integración de estas variables, en un modelo que permite ver la interacción entre las mismas, y cómo éstas de manera directa e indirecta permiten predecir la satisfacción laboral. De este modo, el modelo indica que la probabilidad de que la persona se sienta más satisfecha con su trabajo aumentará a medida que aumente su percepción de competencia en relación a sus tareas laborales, tenga expectativas favorables de resultado, perciba progreso sobre las metas laborales, reciba apoyo de su ambiente, y posea una predisposición a experimentar afectos positivos. Asimismo, dadas todas estas condiciones, es más probable que la persona se sienta satisfecha con su vida (Lent et al. 2011).

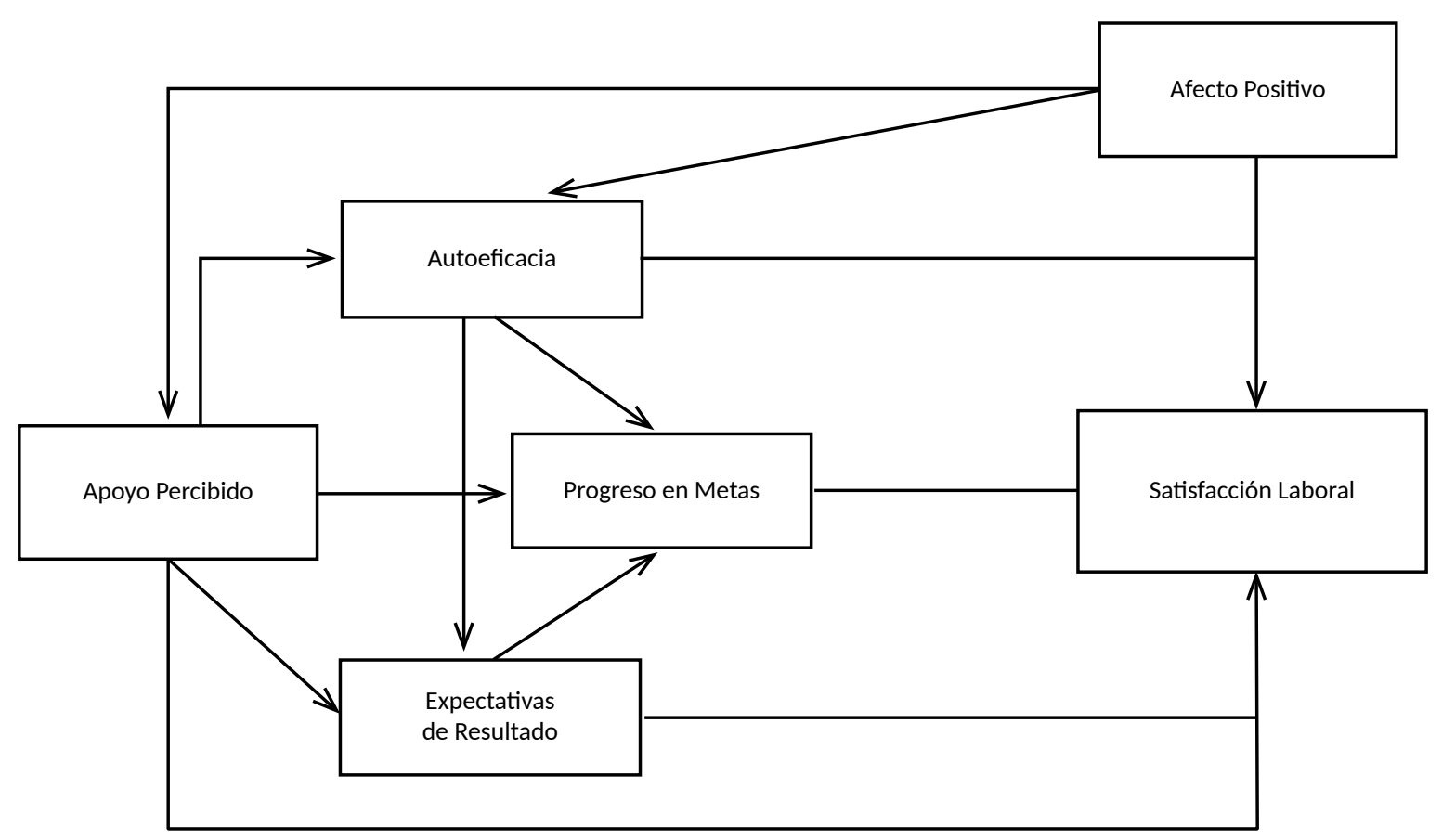

Figura 1. Modelo Social Cognitivo de Satisfacción Laboral y Satisfacción con la Vida (adaptado de Lent, 2004).

Sumado al modelo general, es posible también verificar la relación entre los factores precursores de la satisfacción laboral. Así, por ejemplo, el afecto positivo presenta una relación directa y positiva con la autoeficacia, la satisfacción laboral, la satisfacción con la vida y la percepción de apoyo. Es esperable de este modo que aquellas personas que experimenten altos niveles de afecto positivo evalúen de manera más positiva su autoeficacia y el apoyo percibido (Lent \& Brown, 2006). Cada uno de estos paths ha sido extensamente desarrollados en los estudios de Lent et al. (2011), Lent y Brown (2006) y Badri, Mohaidat, Ferrandino y Mourad (2013).

Diversas investigaciones se han llevado a cabo para verificar la adecuación de este modelo (Badri et al., 2013; Duffy \& Lent, 2009; Farzaneh, Mojtaba, \& Mohammad, 2013; Lent et al., 2011). Lent et al. (2011) llevaron a cabo un estudio del que participaron un total de 235 profesores de Italia. En el mismo se encontraron adecuados índices de ajustes según los criterios
SRMR (.04) y CFI (.98), aunque el valor RMSEA no fue óptimo (.10). El modelo logró explicar un $41 \%$ de la varianza de la satisfacción laboral y un $24 \%$ de la satisfacción con la vida. Con respecto a las relaciones entre los distintos predictores, se encontraron relaciones no significativas en el path desde la percepción de apoyo a la autoeficacia. Similarmente, el path entre el progreso en metas y la autoeficacia tampoco fue significativo. Si bien se comparó el ajuste del modelo sin contemplar los path no significativos, los cambios en la mejora del ajuste no fueron estadísticamente significativos.

Por su parte, Duffy y Lent (2009) trabajaron sobre una muestra de 366 maestros de Carolina del Norte. El modelo dio cuenta de un ajuste óptimo ( $C F I=.97$; RMSEA $=.05$ ). Además, el modelo permitió explicar el $75 \%$ de la varianza de la satisfacción con el trabajo. Sin embargo, ciertos path propuestos no fueron estadísticamente significativos. Entre estos, no se corroboró el path entre la percepción de apoyo y la autoeficacia, ni 
entre la percepción de apoyo y la satisfacción laboral. Asimismo, el progreso en metas no demostró estar relacionada a la satisfacción laboral.

Badri et al. (2013), también indagaron la adecuación del modelo. El mismo fue testeado en 5022 docentes de Abu Dhabi. El modelo demostró un óptimo ajuste $\left(\chi^{2}[4]=14.69, p<0.0538, \mathrm{CFI}=1.0, \mathrm{RMSEA}=0.023\right.$, SRMR $=0.0048$ ) explicando un $82 \%$ de la varianza de la satisfacción laboral. Así, en general el modelo de ecuaciones estructurales corrobora la implicancia de los predictores de la satisfacción con el trabajo y la mayoría de los path propuestos. Sin embargo, no se encuentran paths significativos entre la percepción de apoyo y el progreso en metas; ni entre la percepción de apoyo y la satisfacción laboral; así como tampoco entre la autoeficacia y la satisfacción laboral. Similarmente, Farzaneh, et al. (2013), encontraron resultados similares al estudiar el ajuste del modelo en 382 maestros de secundaria. Los autores obtuvieron índices de ajustes adecuados $\left(x^{2} / d f<3\right.$; RMSEA $\left.=.07\right)$ encontrando que todos los path del modelo eran positivos y estadísticamente significativos.

En un meta-análisis realizado por Sheu y Bordon (2016) se analizaron 37 trabajos donde se analizaba la adecuación del modelo social cognitivo de la profesión propuesto por Lent et al. (2011). Los resultados del trabajo mostraron índices ópimos de ajuste para el modelo evaluado.

A pesar de las anteriores evidencias del ajuste del modelo de Lent, no se encuentran investigaciones llevadas a cabo en el contexto argentino ni sudamericano, en donde se haya testeado el ajuste del modelo propuesto. De hecho, en un meta-análisis hecho por Sheu y Bordon (2016) se analizaron trabajos hechos en poblaciones no estadounidenses y se consideraron trabajos realizados en Europa, Asia, Oceanía y África, pero ningún trabajo del contexto latinoamericano. Sin embargo, en Brasil se ha verificado la asociación de algunas de estas variables sobre la satisfacción laboral (Ramos et al., 2016), no es el modelo de Lent el que se ha indagado, dado que hay un solo eslabón causal en las relaciones propuestas.

En consecuencia, y tal como señalan Lent et al. (2011), para verificar la generalización del modelo es necesario examinar su ajuste en diversas culturas. Esto también coherente con las formulaciones de Lent y Brown (2006), quienes indican que existe poca evidencia acerca del ajuste del modelo propuesto en trabajadores, y que es necesario que se testee en diferentes contextos. Los recientes metanálisis sobre este modelo siguen enfatizando sobre lo mismo, no solo son pocos los estudios en trabajadores, si no que muy poca atención se ha puesto a las influencias culturales (Brown \& Lent, 2017).

El presente trabajo propone analizar dicho ajuste mediante modelos de ecuaciones estructurales en una muestra de trabajadores argentinos). En este sentido, el trabajo se propone aumentar la validez transcultural del modelo (Brown \& Lent, 2017). Es importante y con el fin de ampliar el conocimiento sobre el campo de la satisfacción en el trabajo, que este modelo sea replicado en otros contextos y países para aumentar la validez transcultural del modelo.

\section{Metodología}

\section{Participantes}

Participaron un total de 684 trabajadores ( $90 \%$ del sector privado) de la ciudad de Córdoba, seleccionados por medio de un muestreo no probabilístico de tipo autoelegido. El 55\% de la muestra estaba integrado por hombres y el restante $45 \%$ por mujeres. Los participantes presentaban entre 19 y 67 años de edad $(M=32.8, D E=9.2)$. Respecto al nivel educativo, se observó que la mayor proporción había finalizado sus estudios secundarios o contaba con estudios universitarios incompletos (48\%). El $26 \%$ tenía títulos terciarios o universitarios completos y una proporción menor contaba con estudios primarios completos (5\%). Al considerar las modalidades de contratación se encontró que el $72 \%$ de los trabajadores eran contratados con plazo indeterminado, mientras que el resto de la muestra estaba conformada por trabajadores monotributistas, o que poseían un contrato temporal o se encontraban realizando una pasantía.

\section{Instrumentos}

Escala de Autoeficacia. Para medir la autoeficacia se tomaron los 8 ítems correspondientes al factor ineficacia profesional presentes en el Maslach Burnout Inventory-General Survey (MBI-GS). En este sentido, para obtener una medida de autoeficacia de invirtieron los valores de los ítems correspondientes (e.g. "En mi opinión, soy ineficaz en mi trabajo"). La escala de Burnout de Maslach cuenta con estudios de validez y confiabilidad en Argentina, y el factor correspondiente a ineficacia profesional dio cuenta de un alfa de Cronbach de .67 (Trógolo, Pereyra, \& Sponton, 2013).

Escala de Expectativas de Resultados (Lent, et al., 2007). Compuesta por 10 ítems que indagan 
las consecuencias que perciben los trabajadores al momento de alcanzar una meta (e.g. "si me esfuerzo en el trabajo, conseguiré una promoción/ si cumplo con los objetivos planteados puedo conseguir un aumento"). A los participantes se les presenta una escala Likert de 10 posiciones en donde deben indicar su grado de acuerdo (desde "estoy en fuerte desacuerdo"; hasta "estoy fuertemente de acuerdo"). Se aplicó la versión argentina de la escala, en la cual se verificó una estructura unidimensional así como indicadores adecuados de consistencia interna ( $\alpha=.89, \omega=.89$; Medrano, Ortiz, Flores-Kanter, Dominguez-Lara, \& Gibelli, 2018).

Escala de Apoyo Percibido (Lent et al., 2007). Escala compuesta por nueve ítems desarrollados para evaluar en qué medida el trabajador siente apoyo de su contexto inmediato en la consecución de sus objetivos laborales (e.g. "Tengo la seguridad de que mis compañeros de trabajo tratan de ayudarme/ mis compañeros me apoyan para que cumpla con mis objetivos"). Utilizando una escala tipo Likert, los participantes deben señalar su nivel de acuerdo con cada afirmación (desde "muy en desacuerdo" hasta "totalmente de acuerdo"). Se aplicó la versión argentina de la escala, en la cual se verificó la unidimensionalidad de la escala así como adecuados indicadores de consistencia interna $(\alpha=.84, \omega=.84$; Medrano et al. 2018).

Escala de Progreso en Metas (Lent, Singley, Sheu, Schmidt, \& Schmidt, 2007). Instrumento compuesto por seis ítems que miden el progreso que los trabajadores perciben de sus metas. Para responder, los participantes deben indicar en qué medida han superado cada una de las metas planteadas (e.g. "he logrado cumplir con los plazos estipulados del trabajo/ cumplir con los plazos previstos"), utilizando para esto 10 opciones de respuesta (desde "no he progresado del todo" hasta "he realizado un excelente progreso"). Se aplicó la versión argentina, que da cuenta de valores adecuados de consistencia interna y una estructura unidimensinal ( $a=.85, \omega=.85$; Medrano et al., 2018).

Medición del Afecto Positivo. Se tomo de la Escala de Afecto Positivo y Negativo (PANAS) la sub-escala correspondiente al afecto positivo. La misma fue adaptada en Córdoba, Argentina por Medrano, Flores Kanter, Trógolo, Curarello, y Gonzales (2015). Concretamente, la dimensión de afecto positivo cuenta de 10 ítems que describen distintas emociones positivas, como ser Entusiasmado y Orgulloso, donde la persona debe indicar con qué frecuencia experimenta cada una de ellas utilizando una escala de cinco posiciones. Los resultados de los análisis psicométricos confirmatorios posteriores realizados por Flores-Kanter y Medrano (2016) avalan la confiabilidad de la escala (afecto positivo $a=.86$; afecto negativo $a=.90$ ) y la estructura bidimensional originalmente propuesta para el afecto $\left(X^{2}=547.69, \mathrm{CFI}=.93, \mathrm{TLI}=.92\right.$, RMSEA $\left.=.06\right)$.

Escala de Satisfacción Laboral. En la misma se incluyen siete ítems en los que el examinado evalúa su nivel de satisfacción con diferentes aspectos de su contexto laboral (e.g. "me siento a gusto/ cómodo/ disfruto del ambiente de trabajo/disfruto cuando estoy trabajando"). Para esto se utiliza una escala de 10 posiciones, a través de lo cual los encuestados indican su nivel de acuerdo con cada afirmación. Los estudios psicométricos indican una elevada consistencia interna $(\alpha=.94)$, y una estructura unidimensional (Lent et al., 2007).

\section{Procedimiento}

La muestra se obtuvo mediante un método de muestreo on-line de modo abierto (The International Test Commission, 2006). Esta metodología de recolección de datos ha demostrado ser equivalente a las formas tradicionales de recolección (es decir, cara a cara, Weigold, Weigold, \& Russell, 2013), considerando la equivalencia cuantitativa (es decir, la equivalencia en cuanto a las puntuaciones), la equivalencia cualitativa (es decir, la consistencia interna y las intercorrelaciones) y la equivalencia auxiliar (es decir, las tasas de respuesta y la comodidad de completar cuestionarios utilizando papel y lápiz e Internet). La información fue recopilada a través de la plataforma Google Forms y distribuida a través de redes sociales.

\section{Análisis de Datos}

Para el análisis de los datos, se realizaron primero análisis exploratorios. Se realizaron para verificar la distribución normal univariada y multivariada. La distribución normal univariada se consideró siguiendo los criterios establecidos por Lei y Lomax (2005). Los autores indican que valores de asimetría entre +-1.7 y 1.76 , y de curtosis entre +-3.5 y 3.9 no conducen a sesgos considerables en la estimación de parámetros, utilizando el método de máxima verosimilitud ( $M L)$, ni afectan significativamente los índices de ajustes. Por otra parte, al evaluar la normalidad multivariada, se tomó en consideración el estudio de simulación llevado a cabo por Rodríguez-Ayán y Ruiz-Días (2008), a partir del cual se concluye que un índice de Mardia de kurtosis multivariada $\leq 70$ indicará que la estimación de ML no estará sesgada y proporcionará resultados adecuados. 
El análisis principal de la presente investigación consistió en llevar a cabo un path análisis mediante ecuaciones estructurales. Para evaluar el ajuste de los modelos, se realizó la estimación de los parámetros a través del método de máxima verosimilitud. De acuerdo con la literatura especializada (Garrido, Abad, \& Ponsoda, 2016; Hu \& Bentler, 1999), se utilizó el índice chi-cuadrado $\left(\chi^{2}\right)$, el índice de ajuste comparativo (CFI), el índice de Tucker Lewis (TLI), y la raíz estandarizada media cuadrada residual (SRMR). El SRMR es sugerido por sobre el error cuadrático medio de aproximación (RMSEA), dado que ha demostrado mayor precisión en recientes estudios de simulación (Maydeu-Olivares, Shi, $\&$ Rosseel, 2017). Para la interpretación de los indicadores de ajustes se siguieron los criterios internacionales (Garrido et al. 2016; Hu \& Bentler, 1999; Yu \& Muthén, 2002). Para los índices CFI y TLI, los valores superiores a .90 son aceptables, mientras los valores iguales o superiores a .95 se consideran óptimos. En el caso del SRMR valores inferiores a .08 se consideran indicadores de un buen ajuste. Los análisis mencionados fueron efectuados mediante los softwares estadísticos IBM SPSS 20 y MPLUS 6.12 .

\section{Consideraciones Éticas}

En este estudio se tuvieron en cuenta los lineamientos éticos internacionales al realizar estudios con seres humanos (American Psychological Association, 2017). Al inicio del cuestionario on-line, todos los participantes tenían información respecto a los objetivos de la investigación, el anonimato de sus respuestas, y su participación voluntaria. Asimismo, se aclaró que la participación en la misma no acarrearía ningún daño ni perjuicio para su persona, y que podrían abandonar la encuesta en cuanto lo deseasen. Cabe mencionar que el protocolo de la presente investigación fue previamente aprobado por el comité de ética a cargo de la Secretaría de Investigación de la Universidad Siglo 21.

\section{Resultados}

\section{Análisis Exploratorios Iniciales y Correlaciones}

Se procedió en esta instancia a verificar el supuesto de normalidad univariante y multivariante requerido para este tipo de análisis (Hair, Anderson, Tathan, \& Black, 1999). Respecto a los análisis univariantes, es posible ver en la tabla 1 que los valores de asimetría y curtosis se encuentran en los rangos adecuados. Asimismo, se obtuvo un índice de Mardia de curtosis multivariada dentro de los valores adecuados, siendo igual a 15.75. Todo lo anterior indica que es adecuado llevar a cabo los análisis de ecuaciones estructurales mediante el método de estimación de máxima verosimilitud. En la tabla 1 pueden apreciarse también las correlaciones obtenidas entre las variables incluidas en el modelo. Se obtienen todas correlaciones estadísticamente significativas, en su mayoría de intensidad moderada/alta, en la dirección hipotetizada desde el modelo teórico de base.

Tabla 1. Descriptivos y Correlaciones entre las Variables Incluidas.

\begin{tabular}{lcccccc}
\hline & $\mathbf{1}$ & $\mathbf{2}$ & $\mathbf{3}$ & $\mathbf{4}$ & $\mathbf{5}$ & $\mathbf{6}$ \\
\hline Autoeficacia & 1 & $.44^{* *}$ & $.53^{* *}$ & $.22^{* *}$ & $.64^{* *}$ & $.66^{* *}$ \\
Apoyo Percibido & & 1 & $.47^{* *}$ & $.34^{* *}$ & $.41^{* *}$ & $.58^{* *}$ \\
Expectativas R. & & & 1 & $.37^{* *}$ & $.57^{* *}$ & $.61^{* *}$ \\
Progreso en M. & & & & 1 & $.37^{* *}$ & $.43^{* *}$ \\
Emociones Pos. & & & & & 1 & $.67^{* *}$ \\
Satisfacción L. & & & & & & 1 \\
M & 26.65 & 62.46 & 71.63 & 55.80 & 36.29 & 52.83 \\
DE & 8.35 & 14.80 & 15.86 & 8.09 & 6.88 & 11.95 \\
g1 & -.20 & -.57 & -.65 & -.86 & -.42 & -1.09 \\
g2 & .80 & .03 & .92 & 1.73 & .19 & 1.36 \\
\hline
\end{tabular}

Nota. ${ }^{* *} p<.01 ; M=$ Media; $D E=$ Desviación Estándar; $g 1=$ Asimetría; $g^{2}=$ Curtosis Expectativa R.= Expectativas de Resultado; Progreso en $M=$ Progreso en Metas; Emociones Pos.= Emociones Positivas; Satisfacción L.= Satisfacción Laboral.

\section{Path Análisis: Verificación del Modelo de Lent}

Al llevar a cabo los análisis de la adecuación del modelo, inicialmente algunos de los índices obtenidos indican un ajuste insatisfactorio $\left(X^{2}=9.33, g l=1\right.$, $\mathrm{CFI}=.98, \mathrm{TLI}=.78, \mathrm{SRMR}=.03$ ).

Una de las causas de la mala especificación del modelo puede deberse a la presencia de paths que no presenten una covarianza estadísticamente significativa. Un examen minucioso de los coeficientes de regresión reveló que el path entre autoeficacia y progreso en metas no era estadísticamente significativo ( $\beta$ standarizado $=-.02, p=.80$ ). Al eliminar este path se obtiene un ajuste del modelo óptimo si consideramos los valores CFI y SRMR, y aceptable si se considera el TLI $\left(X^{2}=9.39\right.$, $g \mathrm{I}=2, \mathrm{CFI}=.99, \mathrm{TLI}=.90, \mathrm{SRMR}=.03)$. Los coeficientes estandarizados se muestran en la figura 2. 


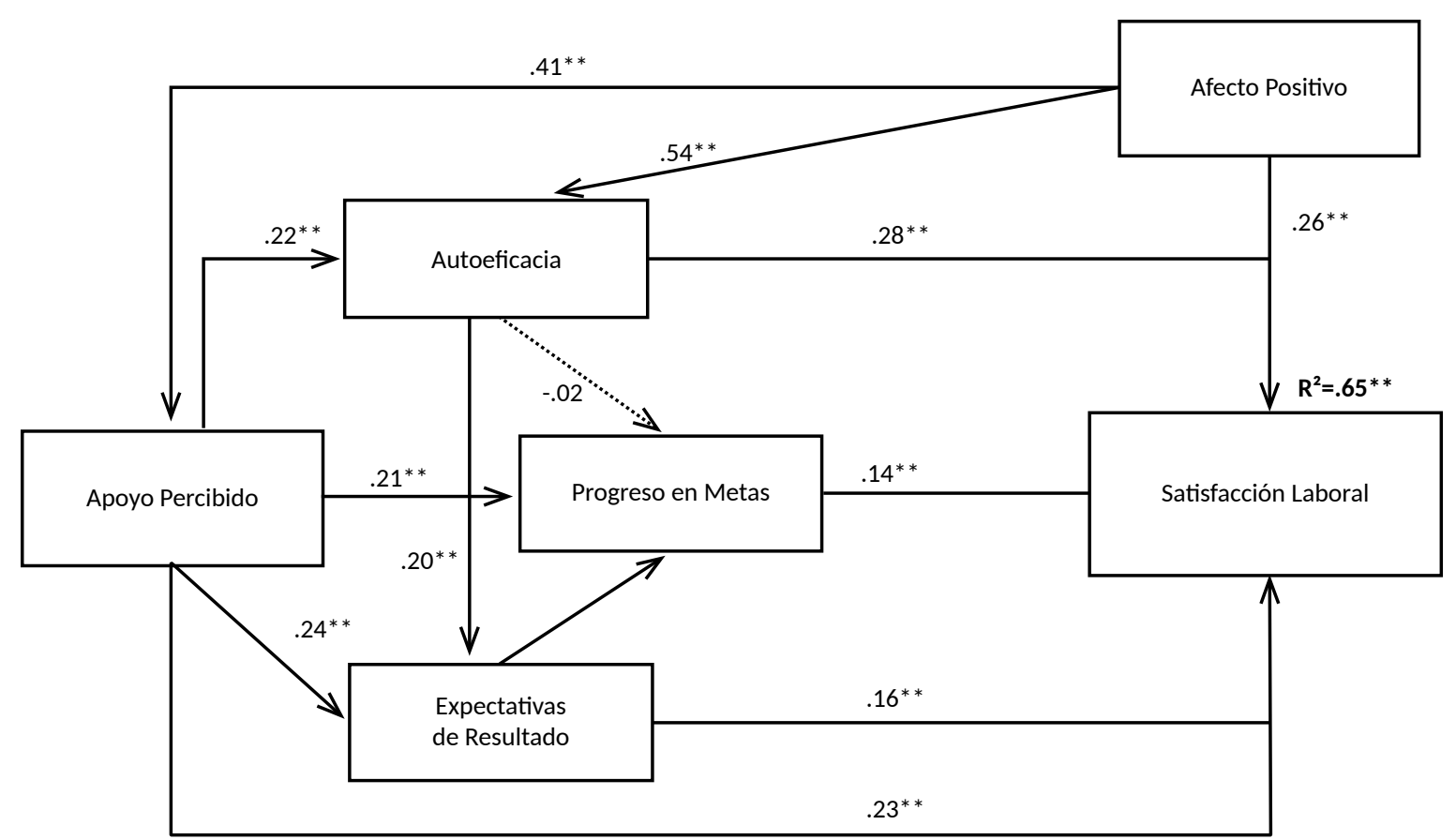

Figura 2. Modelo Social Cognitivo de Satisfacción Laboral y Satisfacción con la Vida (adaptado de Lent, 2004). Visualización de Los Coeficientes Estandarizados.

Nota: ${ }^{* *} p<.01$. La línea discontinua entre Autoeficacia y Progreso en Metas da cuenta del path eliminado ( $\beta$ standarizado $=-.02, p=.80$ ) para un mejor ajuste del modelo.

Finalmente, al examinar la magnitud de los efectos totales estandarizados, se observa que las variables que más contribuyen a la satisfacción laboral son el afecto positivo $(\beta$ total $=.66)$ y el apoyo percibido $(\beta$ total $=.38$ ). Siguiendo en orden de magnitud les siguen la autoeficacia $(\beta$ total $=.31$ ), las expectativas de resultado $(\beta$ total $=.20)$ y el progreso en metas $(\beta$ total $=.15)$.

\section{Discusión}

El presente trabajo se inserta dentro de las líneas de investigación que se interesan por indagar los factores que permiten predecir la satisfacción laboral (Duffy \& Lent, 2009; Lent et al., 2011). El objetivo central fue verificar la adecuación del modelo social cognitivo propuesto por Lent (2004) en trabajadores argentinos.

En general, los resultados muestran que este modelo presenta un ajuste adecuado. En efecto, las variables incluidas en el modelo explican el $65 \%$ de la variabilidad de la Satisfacción Laboral. Asimismo, la mayoría de los path sugeridos por este modelo hacen una contribución significativa a la satisfacción, tanto directa como indirectamente.

Estos hallazgos son similares a los verificados por Lentetal. (2011), Duffy y Lent, (2009), Farzanehetal. (2013) y Badri et al. (2013). Así, en todos estos estudios se verificaron proporciones de varianza explicadas de la satisfacción laboral similares a las del presente estudio (valores $R^{2}$ entre .41 y .82), así como índices de ajuste satisfactorios.

Por otra parte, no todos los paths propuestos demuestran relaciones estadísticamente significativas en los antecedentes indagados. En nuestro estudio se encontró la misma situación que en Lent et al. (2011), siendo el path entre las creencias de autoeficacia y el progreso en metas no estadísticamente significativo. Esta situación también se verifica en el meta-analisis realizado por Sheu y Bordon (2016), que indican una relación pequeña entre el progreso en metas y la autoeficacia.

Sumado a lo anterior, en el presente estudio se verificó que las variables que mejor permiten predecir la satisfacción laboral son, el afecto positivo y el apoyo percibido. En el meta-analisis realizado por Sheu y Bordon (2016) se verifica esta situación, siendo el afecto positivo un factor que influye directa e indirectamente sobre la satisfacción en el trabajo.

Por su parte, la implicancia del apoyo percibido sobre la satisfacción sugiere además que no solo los afectos disposicionales como el afecto son relevantes para predecir la satisfacción, sino que también son de 
particular importancia aspectos del ambiente organizacional, como lo es el apoyo brindado por el contexto de trabajo (Lent, 2013).

Resulta de relevancia indicar las limitaciones que ha presentado este trabajo. Por un lado, se ha trabajado con un tamaño muestral muy pequeño, que imposibilitó realizar análisis de invarianzas tan importante al testear este tipo de modelos (Byrne, 2010). Futuras investigaciones deberían corroborar el ajuste de este modelo, considerando variables sociodemográficas de relevancia como ser el sexo y la edad. Otra de las limitaciones ha remarcar tiene que ver con el diseño utilizado. En efecto, el tipo de diseño utilizado no permite extraer conclusiones definitivas sobre la causalidad o antecedencia temporal de las variables utilizadas en el modelo. La utilización de diseños longitudinales en futuras investigaciones permitirá hacer inferencias más rigurosas con respecto a las relaciones causales involucradas (Cole \& Maxwell, 2003).

Más allá de las anteriores limitaciones, la presente investigación ha presentado por primera vez evidencia del modelo social cognitivo de satisfacción laboral en un contexto sudamericano. Lo anterior es de importancia en dos sentidos. Por un lado, pudo verificarse en gran parte el modelo propuesto por Lent (2004) en un contexto diferente del cual originalmente fue propuesto, lo que aporta a la generabilidad del modelo (Lent \& Brown, 2006). Por otra parte, siendo la satisfacción laboral una meta a alcanzar para lograr un mayor bienestar en los trabajadores, más aún considerando su relación con la satisfacción con la vida (Lent et al., 2011), este estudio da cuenta de aquellas variables que permiten explicar en gran medida su ocurrencia, posibilitando planificar intervenciones enfocadas en cada uno de estos factores. Similar a lo que plantean Lent y Brown (2006), y considerando los resultados obtenidos al verificar el ajuste del modelo, pueden proponerse medidas preventivas, de promoción, o de solución para situaciones de insatisfacción laboral. De esta forma, por ejemplo, intervenciones que se ocupen de lograr un mayor nivel de afecto positivo y mayor soporte institucional a los trabajadores, lograran a su vez un cambio grande en la satisfacción que estos tengan con su trabajo, debido a que el cambio ejercido sobre estas variables repercutirá no solo directamente sobre la satisfacción laboral, sino que, además, generará una modificación en otros recursos personales socio-cognitivos de relevancia (Medrano et al., 2018), como ser la autoeficacia, las expectativas de resultado, o el progreso en metas. (Maffei, Spontón, Spontón, Castellano, \& Medrano, 2012).

\section{Referencias}

American Psychological Association. (2017). Ethical principles of psychologists and code of conduct. Washington, EEUU: Autor. Disponible en http://www.apa.org/ethics

Badri, M. A., Mohaidat, J., Ferrandino, V., \& Mourad, T. E. (2013). The social cognitive model of job satisfaction among teachers: Testing and validation. International Journal of Educational Research, 57, 12-24. doi: 10.1016/j.jijer.2012.10.007

Bandura, A. (Ed.) (1999). Auto-eficacia: cómo afrontamos los cambios de la sociedad actual. España: Desclée de Brouwer.

Brown, S. D., \& Lent, R. W. (2017). Social cognitive career theory in a diverse world. Journal of Career Assessment, 25(1), 173-180. doi: $10.1177 / 1069072716660061$

Byrne, B. M. (2010). Structural equation modeling with AMOS. Basic concepts, applications, and programming. Nueva lorque: Routledge.

Cole, D. A., \& Maxwell, S. E. (2003). Testing mediational models with longitudinal data: Questions and tips in the use of structural equation modeling. Journal of Abnormal Psychology, 112(4), 558-577. doi:10.1037/0021-843x.112.4.558

Duffy, R. D., \& Lent, R. W. (2009). Test of a social cognitive model of work satisfaction in teachers. Journal of Vocational Behavior, 75, 212-223. doi:10.1016/j.jvb.2009.06.001

Farzaneh, M. M., Mojtaba, A., \& Mohammad, H. (2013). Test of a social cognitive model of job satisfaction in urmia secondary school teachers (Abstract). Journal of Educational Innovation, 11(44), 7-13. Disponible en https://www.sid.ir/en/journal/NiewPaper.aspx?ID=291485

Flores Kanter, P.E., \& Medrano, L. A. (2016). El afecto y sus dimensiones: contrastes de modelos ortogonales y oblicuos mediante análisis factorial confirmatorio de la Escala PANAS. Revista LIBERABIT, 22(2), 173-184. Disponible en http://revistaliberabit.com/es/revistas/ RLE_22_2_el-afecto-y-sus-dimensiones-modelos-contrastadosmediante-analisis-factorial-confirmatorio-de-la-escala-panas.pdf

Garrido, L. E., Abad, F. J., \& Ponsoda, V. (2016). Are fit indices really fit to estimate the number of factors with categorical variables? Some cautionary findings via Monte Carlo simulation. Psychological Methods, 21(1), 93-111. doi: 10.1037/met0000064

Hair, J. F., Anderson, R. E., Tatham, R. L., \& Black, W. C. (1999). Análisis Multivariante. Madrid: Person Prentice Hall.

Hu, L., \& Bentler, P. M. (1999). Cutoff criteria for fit indexes in covariance structure analysis: Conventional criteria versus new alternatives. Structural Equation Modeling: A Multidisciplinary Journal, 6(1), 1-55. doi: 10.1080/10705519909540118

Lei, M., \& Lomax, R. G. (2005). The effect of varying degrees of nonnormality in structural equation modeling. Structural Equation Modeling: A Multidisciplinary Journal, 12(1), 1-27. doi: 10.1207/s15328007sem1201_1

Lent, R.W. (2004). Toward a unifying theoretical and practical perspective on well-being as psychosocial adjustment. Journal of Counseling Psychology, 51(4), 482-509. doi: 10.1037/0022-0167.51.4.482

Lent, R.W. (2013). Career-life preparedness: Revisiting career planning and adjustment in the new workplace. The Career Development Quarterly, 61(1), 2-14. doi: 10.1002/j.2161-0045.2013.00031.x 
Evaluación de un modelo socio-cognitivo de la satisfacción laboral en trabajadores argentinos

Lent, R.W., \& Brown, S.D. (2006). On conceptualizing and assessing social cognitive constructs in career research: A measurement guide. Journal of Career Assessment, 14(1), 12-35. doi: 10.1177/1069072705281364

Lent, R. W., \& Brown, S. D. (2008). Social cognitive career theory and subjective well-being in the context of work. Journal of Career Assessment, 16, 6-21. doi: 10.1177/1069072707305769

Lent, R. W., Nota, L., Soresi, S., Ginevra, M. C., Duffy, R. D., \& Brown, S. D. (2011). Predicting the job and life satisfaction of Italian teachers: Test of a social cognitive model. Journal of Vocational Behavior, 79, 91-97. doi: 10.1016/j.jvb.2010.12.006

Lent, R. W., Singley, D., Sheu, H. B., Schmidt, J. A., \& Schmidt, L. C. (2007). Relation of social-cognitive factors to academic satisfaction in engineering students. Journal of Career Assessment, 15(1), 87-97. doi: 10.1177/1069072706294518

Liu, H., Zhang, X., Chang, R., \& Wang, W. (2017). A research regarding the relationship among intensive care nurses' self-esteem, job satisfaction and subjective well-being. International Journal of Nursing Sciences, 4(3), 291-295. doi: 10.1016/j.ijnss.2017.06.008

Maffei, L., Spontón, C., Spontón, M., Castellano, E., \& Medrano, L. A.(2012). Adaptación del Cuestionario de Autoeficacia Profesional (AU-10) a la población de trabajadores cordobeses. Pensamiento Psicológico, 10(1), 51-62. Disponible en http://www.scielo.org.co/scielo. php?script=sci_arttext\&pid=S1657-89612012000100004\&lng $=e$ n\&ting=es

Maydeu-Olivares, A., Shi, D., \& Rosseel, Y. (2017). Assessing fit in structural equation models: A Monte-Carlo evaluation of RMSEA versus SRMR confidence intervals and tests of close fit. Structural Equation Modeling: A Multidisciplinary Journal, 25(3), 389-402. doi:10.1080/10705511.2017.1389611

Medrano, L. A., Flores Kanter, P.E., Trógolo, M., Curarello, A., \&. Gonzales, J. (2015). Adaptación de la Escala de Afecto Positivo y Negativo (PANAS) para la población de Estudiantes Universitarios de Córdoba. Anuario de Investigaciones de la Facultad de Psicología, 2(1), 22-36. Disponible en https://revistas.unc.edu.ar/index.php/aifp/article/ download/12503/12773

Medrano, L. A., Ortiz, A., Flores-Kanter, P. E., Dominguez-Lara, S., \& Gibelli, S. (2018). Medición de recursos personales socio-cognitivos en organizaciones: Análisis psicométricos en trabajadores argentinos. Revista Evaluar, 18(2), 01-16. Disponible en https://revistas.unc.edu. ar/index.php/revaluar/article/view/20799

Ramos, M. F. H., Fernandez, A. P. O., Furtado, K. C. N., Ramos, E. M. L. S., Souza, S. S. C. C., \& Ramos Pontes, F. A. (2016). Satisfação no trabalho docente: uma análise a partir do modelo social cognitivo de satisfação no trabalho e da eficácia coletiva docente. Estudos de Psicologia 21(2), 179-91. doi: 10.5935/1678-4669.20160018

Rodríguez-Ayán, M. N., \& Ruiz-Díaz, M. A. (2008). Atenuación de la asimetría y de la curtosis de las puntuaciones observadas mediante transformaciones de variables: Incidencia sobre la estructura factorial Psicológica, 29, 205-227. Disponible en <http://www.redalyc.org/ articulo.oa?id=16929206>

Sanjuán Suárez, P., \& Rueda Laffond, B. (2014). Promoción y prevención de la salud desde la psicología. Madrid: Síntesis Editorial

Sheu, H.-B., \& Bordon, J. J. (2016). SCCT research in the international context. Journal of Career Assessment, 25(1), 58-74. doi: $10.1177 / 1069072716657826$

Spector, P.E. (1997). Job satisfaction: Application, assessment, causes, and consequences. Thousando Oaks, California: Sage publications.

The International Test Commission. (2006). International guidelines on computer-based and internet-delivered testing. International Journal of Testing, 6(2), 143-171. doi: 10.1207/s15327574ijt0602_4

Trógolo, M., Pereyra, P., \& Spontón, C. (2013). Impacto de diferentes estilos de liderazgo sobre el engagement y burnout: Evidencia en una muestra de trabajadores argentinos. Ciencia \& Trabajo, 15(48), 152-157. doi: 10.4067/S0718-24492013000300008

Weigold, A., Weigold, I. K., \& Russell, E. J. (2013). Examination of the equivalence of self-report survey-based paper-and-pencil and internet data collection methods. Psychological Methods, 18(1), 53-70. doi: 10.1037/a0031607

Yu, C.Y., \& Muthen, B. (2002, April). Evaluation of model fit indices for latent variable models with categorical and continuous outcomes. In Annual Meeting of the American Educational Research Association, Nueva Orleans, LA. Disponible en https://www.statmodel.com/download/ Yudissertation.pdf

Lucas Lapuente, licenciado em Psicologia, atua na Universidad Siglo 21, Córdoba/Argentina. Endereço para correspondencia: Universidad Siglo 21. Calle de los Latinos 8555, 5008 Córdoba. E-mail: lucas.lapuente@ues21.edu.ar

Pablo Ezequiel Flores Kanter, licenciado em Psicologia, atua na Universidad Siglo 21, Córdoba/Argentina.

E-mail: pablo.floreskanter@ues21eduar.onmicrosoft.com

Roger Muñoz Navarro, doutor em Psicologia pela Universidad de Valencia/España, é docente na Universidad de Valencia/España.

Leonardo Adrián Medrano, doutor em Psicologia, é docente na Pontificia Universidad Católica Madre y Maestra, Escuela de Psicología, Republica Dominicana. E-mail: leonardo.medrano@ues21.edu.ar

Recibido en 03.mayo.18 Reformulado en 11.abr.19 Aceptado en 16.jul.19

Estudos de Psicologia, 24(1), janeiro a março de 2019, 12-20 